\title{
A Modular Approach to Promote Creativity and Inspiration in Search
}

\author{
Alice Thudt \\ InnoVis Group, University of \\ Calgary, Canada \\ alice.thudt@gmail.com
}

\author{
Uta Hinrichs \\ SACHI Group, University of \\ St Andrews, UK \\ uh3@st-andrews.ac.uk
}

\author{
Sheelagh Carpendale \\ InnoVis Group, University of \\ Calgary, Canada \\ sheelagh@ucalgary.ca
}

\begin{abstract}
When searching through collections of books or written texts, the efficient yet limiting query paradigm is still the most dominant entry point. Previous work characterizes search processes in various contexts and describes them as integral and closely related to creative endeavours. We revisit this work from a design perspective, proposing guidelines for versatile search interfaces that are based on a modular approach to search. Inspired by aspects of search in physical environments, our recommendations address learning, creativity, inspiration, and pleasure as positive aspects of (book) search. Based on in-depth interviews with library patrons about search practises in physical and digital environments and drawing from previous work on search behaviour, we discuss search patterns as modular constructs consisting of micro-strategies. We illustrate how the structure of these patterns is highly flexible. Much like creative processes, they fluidly evolve based on learning and ideation during search, particularly in physical environments. This modular perspective provides a basis for designing interfaces that facilitate creative approaches to search in digital environments.
\end{abstract}

\section{Author Keywords}

Book Search; Creativity; Search Interface; Qualitative Study

\section{ACM Classification Keywords}

H.5.m. Information Interfaces and Presentation (e.g. HCI): Miscellaneous

\section{INTRODUCTION}

With the transition of many information services to the digital realm, search is no longer just the domain of knowledge workers-it directly impacts everyday life. Search interfaces have become the go-to point for finding books, articles, recipes, and people with shared interests, and also, for inspiration and ideation. This has led to expanding interest in the nature of search from a wide variety of fields.

Research in library and information sciences has recognized search as a complex and fluid process that cannot be satisfied by simple query-response paradigms $[3,28,47,53]$. A large

Permission to make digital or hard copies of all or part of this work for personal or classroom use is granted without fee provided that copies are not made or distributed for profit or commercial advantage and that copies bear this notice and the full citation on the first page. Copyrights for components of this work owned by others than the author(s) must be honored. Abstracting with credit is permitted. To copy otherwise, or republish, to post on servers or to redistribute to lists, requires prior specific permission and/or a fee. Request permissions from Permissions@ acm.org.

$C \& C^{\prime} 15$, June 22 - 25, 2015, Glasgow, United Kingdom.

Copyright is held by the owner/author(s). Publication rights licensed to ACM. ACM 978-1-4503-3598-0/15/06 \$15.00

DOI: http://dx.doi.org/10.1145/2757226.2757253 body of literature has investigated information behaviour in professional $[1,2,3,6,14,31,32]$ and leisure contexts [10, $18,30,42]$. Research has also considered search as part of creative processes $[24,32,46]$ as well as a creative process in itself $[28,29]$. Furthermore, exploratory search [35, 53] and search for pleasure (e.g., [22]) have been discussed, alongside design considerations for versatile interfaces that go beyond targeted search [21, 46, 32, 51, 53, 56].

Despite this, people still predominately face query-based interfaces that hamper the fluid, complex, and creative nature of search endeavours when searching through book collections online. This paper discusses the characteristics of search strategies and processes from a design perspective, bridging insights from information sciences, information retrieval, creativity and design research, and HCI. Similar to Lee et al., we consider search to be a creative process [28, 29] inherent in everyday creativity and individual knowledge construction $[4,23]$. We therefore investigate how knowledge about book search can facilitate the design of interfaces that promote versatile and complex search processes and, as part of this, learning, inspiration, social endeavours, and pleasure.

We interviewed library patrons about their approaches to book search as an activity performed frequently in professional and leisurely contexts, as well as using digital and physical search environments. Participants described a broad range of search behaviours, influenced by the characteristics of the digital or physical information space. They describe their search endeavours as flexible, often complex, search processes that fluidly evolve as learning, ideation, and creative thinking take place. We illustrate how these search processes can be supported through four micro-strategies: querying, linking, scanning, and assessment. While similar strategies have been discussed in previous work [1, 3, 14, 28, 32], we propose these four strategies as the core modules that compose many different forms of book search. We illustrate how considering their composition directly informs the design of flexible search interfaces, merging the advantages of digital and physical search environments. Based on this modular approach to search, we provide guidelines for supporting fluidity in search processes through continuous navigation, search histories, thus promoting sensuous search experiences.

\section{BOOK SEARCH AS A COMPLEX CREATIVE PROCESS}

Search has long been recognized as a special case of problem solving [33], the "problem" being a need for certain information. However, with the increasing predominance of search activities conducted in many contexts ranging from professional to everyday scenarios, recent research has begun to 
consider search and exploration as positive activities that can (and should) be pleasurable in order to promote creative discovery and sensemaking $[12,51]$. In fact, research suggests that search is closely related to creativity and ideation [28, 29, 24] where tactics are fluidly applied, combined, and adjusted in order to reach a certain goal $[1,21,29]$.

Based on our interviews that focused on book search in professional and leisure contexts as well as in digital and physical environments, we describe how flexible and creative search processes are formed by four basic micro-strategies. We discuss implications for design suggested by this modular view on search. Our work builds a vast body of research that has investigated human information behaviour including cognitive and affective aspects, information needs and processes, as well as creativity as it relates to information seeking.

\section{Models of Search Behaviour}

A number of models of search behaviour have been developed based on the analysis of knowledge workers' information seeking (see [21, chpt. 3] for a comprehensive overview). These models can be distinguished based on the goals and granularity in which they classify search behaviours. Some of these models reflect on stages of the iterative process of search, from the identification of an information need, to the query formulation, to the result review [25, 36, 45, 50]. Other research has investigated higher-level strategies $[3,14,25,34$, 32], including, for instance, chaining [14], footnote chasing, and citation searching [3] to find related resources.

Bates introduced one of the earliest facilitation/teaching models for professional reference search that distinguished four categories of low-level tactics with 26 individual tactics in total, including, e.g., correcting the formulation of a search request, surveying possible search terms, or reducing the number of search terms to expand the number of search results [1]. Based on this model, further research investigated search tactics in professional contexts [7, 31, 32, 48, 55]. Similar tactics and strategies are reflected in our research, however, we expand on previous work by exploring search processes from a design perspective. Our work focuses on the flexible formation and composition of micro-strategies which is driven by the search purpose and guided by ideation and learning.

\section{The Context of Search}

Previous research typically distinguishes between professional and leisure search contexts. Professional search is conducted by domain experts and knowledge workers $[1,6,40]$, while leisure search is characterized by personal goals such as pleasure or mastery [22, 30, 39, 42]. A range of literature has investigated professional search behaviours and processes [1, $2,6,9,11,14,17,25,26,31,32]$, e.g., bibliographic and reference search [1], search in digital libraries and on the internet [6, 37, 48], video search [55], and search in creative professions such as architecture [32]. Studies have found that leisurely search, more than professional search, follows personal curiosities $[10,18,20,30]$, however, most research on leisurely information search (e.g., fiction reading) focuses on selection strategies rather than search processes [43, 44, 49]. For instance, while some studies have investigated how people search for fiction books [40, 43, 44, 49], insights do not go beyond broad strategy categories such as "known-item search" or "browsing". Considering both digital and physical search environments, our work contributes the investigation of search processes as they evolve as part of book search in general. We aim at informing the design of search interfaces that can support a large variety of activities as part of both professional and leisurely book search.

While search patterns may differ depending on the context $[40,55]$, our findings indicate that people do not necessarily consciously distinguish between professional and leisure book search. Therefore, we focus on how the characteristics of the search environment (i.e., its digital vs. physical context) influence search and how people experience this as part of their search endeavours.

\section{Search \& Creativity}

Previous research has highlighted parallels between search and creative processes. Shneiderman considers search as part of the creative process and describes how software tools can support creative tasks [46]. Kaufman and Beghetto introduced "mini-c" as an aspect of creativity that focuses on "the dynamic, interpretative process of constructing personal knowledge and understanding" [23, p.3]. They highlighted the relationship between learning and creativity [4]. Information seeking as a process of individual knowledge construction can therefore be considered as an instance of "mini-c". Lee et al. have investigated the relationship between creative and information seeking processes more closely and coined the term "creative information seeking" [28, 29]. Combining models describing the creative process [19] and information seeking behaviour [14], they propose a six-stage model, where individual stages can be combined depending on the complexity of the search task [29]. Expanding on this idea of creativity as a characteristic and driver of information seeking activities [32], we aim at defining micro-strategies as modules of search processes that, through interface design, can be supported and combined in versatile ways and enable opportunities for learning, creativity, and ideation.

\section{Designing Search Interfaces}

Alongside research on search behaviour and its relation to creative processes, a range of considerations on how to design search interfaces exist. Shneiderman argues for visual search interfaces that highlight relations between search results, promote new associations and exploration through filtering, and support search histories [46, 47]. On a more concrete level, Hearst provides general design guidelines for digital search interfaces. Building upon search behaviour models, her guidelines address design aspects such as the importance of supporting versatile queries and query refinement, showing document details within the search result list, highlighting the relation between query terms and search results, and integrating search and the navigation of search results [21]. She also discusses the importance of visual and interaction aesthetics to promote pleasurable and prolonged search activities. Distinguishing four categories of search interface features (input, control, informational, and personalizable features), Wilson provides a feature-centric approach to designing search interfaces [56]. His 26 design recommendations largely overlap with Hearst's guidelines. White and Roth's design recommendations focus on exploratory search interfaces in particular [53]. While several of these recommendations overlap 
with those by Hearst and Wilson, they particularly emphasize the importance of supporting learning and sensemaking through the search interface design to help people tailor and steer their exploration into relevant directions.

While existing design guidelines are fairly concrete when it comes to supporting particular aspects of search such as query refinement, others, such as "supporting learning and understanding", or "offering visualizations to support insight/decision making" [53], remain at a more abstract level. Given the flexible and diverse character of search activities, we propose a new approach to designing search interfaces that aims at supporting versatile search processes by developing design considerations directly from the micro-strategies that form these processes. In this way, a large variety of search scenarios and purposes can be supported, and individual search activities can be dynamically revised and refined as new knowledge and ideas develop during search.

\section{STUDY METHODOLOGY}

Our interview-based study focused on book search as it occurs in both digital and physical environments. Our interview questions were deliberately kept broad and open-ended to tease out people's experiences in different search contexts. For instance, we asked participants about their general approach to book search, that is, activities that they typically apply to find books and textual information for work-related and personal interests, in both digital and physical spaces. We asked them to describe search strategies in detail based on examples, the factors of digital and physical information spaces they appreciated or found annoying, and their general experience and attitude toward search endeavours.

\section{Study Setup \& Participants}

The study was conducted at a university library, where we interviewed patrons about their book exploration habits. Ten library patrons were invited ad-hoc for a spontaneous interview (quoted as A\#, five female). In addition, ten participants were recruited and invited to the library for an interview (quoted as R\#, three female). Recruitment allowed for in-depth interviews and expanded our participant population beyond visitors of the university library. Recruited participants' ages ranged from 20-60 years; six of them were university students from different disciplines and all had used computers for more than five years. No demographic data was collected from library patrons in order to limit their time commitment.

\section{Data Collection \& Analysis}

All 20 interviews were audio recorded and transcribed. The transcripts were coded independently by two researchers following a thematic analysis approach [5]. Two coding passes were applied. In the first pass we coded participants' statements about specific search and exploration approaches, how they manifest in different contexts, and their motivations for book searches. We further coded for experiences and problems in the different (digital and physical) search environments. The codes from this first pass were often quite specific, and were collaboratively analyzed to extract individual strategies, compound processes, and motivations that drive participants' search habits. Through this analysis, higher-level categories emerged: common strategies, including querying, linking, scanning, following recommendations, and reading book previews; desired search outcomes, satisfying a particular reading experience, or locating material on a topic of interest; and complex search processes that are a combination of low-level search strategies.

The resulting final coding schema was applied in a second coding pass. A subsequent analysis resulted in a modular view on book search under the following themes: MicroStrategies, Search Purposes, and Search Processes. We discuss our findings in the following three sections.

\section{FINDINGS}

To set the context for discussing participants' search strategies and processes in-depth, we first report on the general scenarios in which our participants conduct their searches.

Search Contexts. In our interviews we deliberately encouraged a free contemplation of book search. We found that 95\% of our participants described their processes independent of the search context (professional vs. leisure). While participants stated that their search processes may differ depending on the contexts, only one participant clearly separated between professional and personal book search. Our design considerations therefore address both work-related and leisure book search, rather than contrasting them.

Environments. Participants commented on a variety of search environments including public and academic libraries, bookstores and common digital search environments (library catalogues, online book stores, academic and general purpose search engines). While all participants mentioned the use of digital book search engines, only one used exclusively digital environments. Our findings show how micro-strategies and processes manifest in digital and physical environments and highlight their problems and benefits.

In the following three sections we deconstruct search into its basic components. We describe micro-strategies that we identified through our interviews. We outline search and exploration purposes and illustrate in sample scenarios how these purposes drive the fluid composition of micro-strategies into complex search processes. We then discuss the design space that this modular approach to search opens up.

\section{MICRO-STRATEGIES}

There is a large body of work that discussed lower-level activities of information search processes (e.g. [1, 3, 14, 25]). As part of this work, different terminologies were used. Bates defines the term search tactics, i.e., low-level moves that are applied to further and revise a search [1]. The terms "search tactic" and "moves" have been used relatively consistently to describe low-level search activities $[48,55]$, however, Fidel introduces "moves" as part of search tactics and strategies [15]. The term search strategy is used in various ways, sometimes for the search as a whole [1] and sometimes to describe activities that are less specific than search tactics, but are still at a relatively low level of granularity [3,14] (e.g., area scanning, author searching, and citation searching as search strategies [3]). While some discussions of search strategies suggest their sequential character [25], others highlight that, depending on the search tasks, they can be combined in different ways, and often iteratively [14, 28, 29]. 
Our interviews revealed search strategies that are similar to the ones discussed in previous literature. We define microstrategies as components from which a search process is composed. Micro-strategies are (1) abstract and thus apply to a variety of scenarios, (2) consistent in their level of granularity, and (3) sufficiently descriptive to imply interface design guidelines. We describe these micro-strategies in the light of previous literature and emphasize how each of them is supported in digital and physical environments - an aspect that our interview analysis contributes to the field.

QUERYING, i.e, issuing an inquiry (e.g, a question, keyword, title, or author name) to a system, person, or other point of reference was mentioned by all participants. This does not come as a surprise-querying as part of search has been extensively discussed in previous literature [1, 8, 34, 35]. Most digital search interfaces support querying well: typing a term into a text field produces a list of results. Querying can be further facilitated through design, e.g., by suggesting related terms (see [21, chpt.1] for more details). Seven of our participants also described querying in bookstores or libraries by asking a staff member to help them find a book or section.

$\rightarrow$ Queries for specific books are typically performed by title and/or author. $5 \bigcirc \begin{aligned} & \text { Less targeted queries by topic or keyword can } \\ & \text { produce much larger result sets. }\end{aligned}$

LINKING describes strategies in which the information seeker is led from a "seed item" to one or more related items. Kwasnik describes digital browsing as "movement in a connected space" [26] guided by links. This can be either itemto-item linking between individual books, or item-to-set linking, connecting a single book to a book collection.

Item-to-item linking has been discussed, e.g., as forward and backward chaining [14, 28] and citation searching and footnote chasing [3]. Participants' statements confirm that citation linking is applied digitally and physically. People also follow other links between individual books in a physical space by browsing adjacent books on a bookshelf. Digitally, recommender systems can provide item-to-item links based on peoples browsing or purchasing behaviour.

In item-to-set linking, an individual book leads to a set of books, for instance, by the same author or about a related topic. In physical environments the location of a specific book on a shelf can be considered as a connection to related adjacent material. Our participants mentioned the use of a variety of such linking features in digital search environments, for example, the use of author and keyword links to find related books sets.

SCANNING describes strategies in which a set of books is skimmed visually or through physical movement to gain an overview in preparation for selecting particular items. In contrast to assessment strategies (see next paragraph), scanning focuses on visual aspects of books that are readily available without further interaction (e.g., title and author visible on the book spine or in a digital result list). For example, the book's title alone can provide an idea about its topic to enable quick pre-selection. Besides textual features, visual and aesthetic characteristics of books can act as eye catchers. Participants also mentioned an attraction to books that evoke personal associations or visually stood out from the rest of the collection. They highlighted the importance of the spatial layout of items to allow taking in their visual cues, and particularly lauded physical environments for their visual richness.

Scanning has been discussed in the context of browsing [9, $14,34]$, e.g., when trying to locate a known book in a collection, (linear and selective scanning as part of search browsing [34]), getting an overview of a collection (semi-directed or general purpose browsing [34]), or for more open-ended explorations (serendipity or casual browsing [34, 35]). Area scanning has been referred to as an activity in which the searcher looks at adjacent books [3].

ASSESSMENT describes strategies in which an individual item is inspected for its relevance to the search purpose. Similar strategies have been previously discussed as differentiating, evaluation or exclusion/negation, in which particular items are ruled out [35], or selecting, in which useful items are chosen [31]. Our participants frequently mentioned assessment strategies as part of their book search. In contrast to scanning (gaining an overview and identifying books of potential interest, see above), assessment strategies include a qualitative judgement of individual items to make an informed choice. While Makri discusses assessment as a highlevel strategy that includes selecting, distinguishing, browsing, and extracting [32], we consider assessment as a microstrategy that can include meta-level and content-level investigation of found items, similar to what Buchanan and McKay discuss as "closed book" and "open book" assessment [8].

Meta-level assessment is based on a book's metadata (e.g., title, author, abstract or citation rank). When using digital search interfaces, participants stated that they assess items based on their position in a result list, where the ordering of search results acts as a measure for relevance of the book content. Participants further mentioned that the "look and feel" of a book influences if they acquire it or not. In particular, physical criteria can hint at a particular reading experience.

Content-level assessment considers the book's

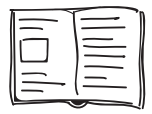
content or writing style, e.g. by skimming through it. While physical books provide direct access to their content, book previews provided by some digital platforms fulfill a similar purpose. Reading reviews is another content-level assessment strategy. Participants particularly value reviews if selection criteria are difficult to assess through the book's metadata.

Scanning and assessment can be considered as components of what Marshall and Shipman have coined as "information triage [37]. With our design-oriented approach to deciphering search in mind, we aimed at providing more specific distinctions between different strategies that fall into this category. 


\section{SEARCH \& EXPLORATION PURPOSES}

Previous research has suggested that search can consist of fluid, sometimes iterative processes depending on the search purpose [29]. Our research expands on this by illustrating the diversity and complexity of such processes based on our micro-strategies. Our findings confirm that book explorations cover a range of purposes beyond well-defined information needs, as indicated in previous research on exploratory search and everyday life information seeking [8, 22, 35, 39, 53]. Specifically, we identify four search purposes that go beyond finding a known book: finding books within constrained criteria, discovery of new topics, knowledge and understanding, and a rewarding, inspirational exploration experience.

\section{Constrained Criteria}

Similarly to Buchanan and McKay [8], we found searches for new books within more or less specific criteria to be common in both leisure and professional contexts. The relation to a known book can help to specify the nature of the desired book: "I want a book that is related to this [known book] but I don't know exactly what I want." [R6]. Library classification systems support this purpose by organizing books based on content similarity. Searches with broader criteria (e.g. topic or genre) are experienced as more difficult, especially with common query approaches that can lead to an abundance of results: "If I was looking for something like non-academic literature it is hard. I get all kinds of websites, non-relevant searches and stuff, just because a keyword overlaps. I can't read in there, it's too much. [...] I give up." [A11].

Participants also described searches for books that encompass a particular reading experience. Such criteria are particularly difficult to express in a query and our participants experienced these searches as cumbersome in digital environments: "Let's say I want to read a book for pleasure, but where do I start? Which author? What language? What subject?" [A8].

\section{Topic Discovery}

An even more open-ended purpose of book search is the discovery of books on new, unknown topics. Here, the purpose is to be inspired; to identify new topics of interest and to discover related books: "I' $m$ really enthusiastic to find that kind of untouched areas.” [R3]. The driver of such open-ended exploration is a general "curiosity, I guess, or just thirst for more. I don't know, I just want to keep discovering." [R10].

Participants described such open-ended explorations for "something different" as better supported in physical libraries or bookstores than in digital search interfaces: "I really didn't know what keywords I should put in to find what I wanted, because the thing is, I really didn't know exactly what I wanted. So this is the problem-if you know, [what you are looking for] it's easy, but if you don't, if you just want to read something different, it's kind of hard." [R6]. Participants bemoaned a loss of opportunities for open-ended explorations and serendipitous discoveries with the transition of book collections to the digital realm: "So that kind of sense of digging through the shelves, looking for hidden treasures [...] seems to be just going away." [R10].

\section{Knowledge \& Understanding}

Some book explorations that participants described follow the purpose of gaining an understanding or furthering knowledge about a particular topic, rather than locating one or multiple books. In these searches, books become a portal to the desired information but are not the actual target of the search: "I would also like to have a digital copy, so I could search for things [within the book] easily, to find stuff that I'm looking for." [R6]. The focus is on the information bits hidden within books, not necessarily on the books themselves.

Another search outcome may also be information toward a broader question: "I have my research question. That is my query, and then I always have a thing I want to prove in mind. [...] And then I go with that book that really fits, what I want to prove. And then I go and I'll say: 'Oh this would be really great backup information or this would be something to add to it." ' [R7]. Physical books lend themselves to quickly getting a content overview and, hence, to learn about a topic: "When you have it in your hand, it is easier to flip through it, it's like you can [adjust] the speed or the slowness with which you go through it. [...] Whereas online previews are like clicking one page, one page, one page.” [R5].

\section{Exploration Experience \& Inspiration}

Our interviews also pointed to a form of book exploration where the purpose is neither acquisition of books nor furthering knowledge. Instead, the exploration experience can be rewarding in itself, especially if the environment or collection is inviting: "For the joy, yes. I do that [going to a bookstore] for the joy." [R4]. "So just finding a whole group of them [comics] and just being 'Oh wow, let's go through all these..., yeah, there's something to that." [R10].

Participants usually turned to physical environments such as bookstores when aiming for a rewarding stroll through a book collection: "Sometimes I wish libraries were kind of more like bookstores. If you go into [bookstore name] you'll always see signs saying 'Adult Fiction'. [...] In academic libraries I feel like, sometimes I go in there and it's always just call numbers. And that's great for librarians, because they know what that means, but I'm just a user, so I don't know what that means." [R7]. The focus on efficiency in digital interfaces neglects the support of exploration solely for "joy" [R4] as the following statements points out: "I guess the idea of like a shopping expedition to go look for something, like that kind of fun is gone. But I guess it's more efficient to just have everything at your fingertip at home." [R10].

\section{CONSTRUCTION OF COMPLEX SEARCH PROCESSES}

The purposes described above drive the combination of micro-strategies into search processes. Our interviews indicate the fluidity and complexity of some search processes where even the initial purpose can change on the fly. In the following, we illustrate the construction of a range of processes from micro-strategies according to scenarios extracted from our interviews. We further discuss learning, social aspects and pleasure as concepts that are an integral part of search as a creative endeavour.

\section{Process Composition by Example}

The exploration processes we extracted from our interviews indicate a range of complexity. Targeted search purposes ( $\rightarrow$ Known Book or Constrained Criteria) are based 
on straightforward processes with a simple combination of micro-strategies. The more open-ended the purpose of a book search ( $\rightarrow$ Topic Discovery or Knowledge \& Understanding), the more complex and iterative the search process becomes.

Known-book search is the simplest type of search and often accomplished by querying. Targeted search processes with the purpose of satisfying Constrained Criteria typically require a combination of micro-strategies as illustrated in Scenario 1: "When I read in the train, I really know what I want to read, and I read almost every time the same kind of book. So I know which author I like, or which kind of book I want. [...] I pick the book because I know the author, or it's just a new best-seller. I pick the book, I read the summary. Probably, I look into the book on two or three pages, if I like how the author writes, and then I buy it or not." [R9].

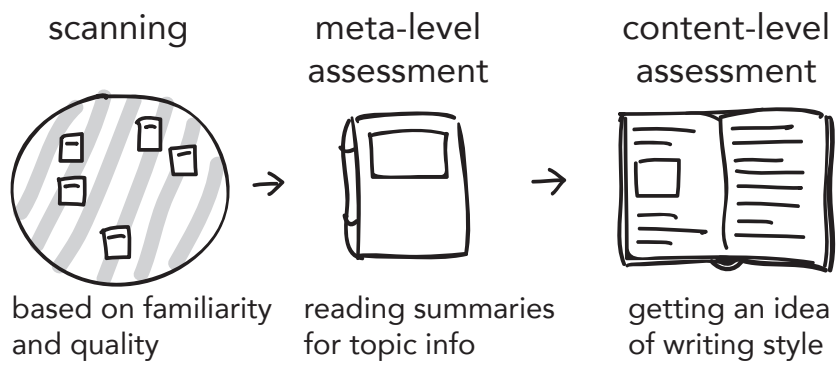

Scenario 1. Finding books within certain criteria.

This participant has a clear idea of the type of book she wants to read in a particular context (the train) without having a particular title in mind. She first scans available sources for books that promise a certain reading experience. Familiarity drives her scanning activity as she has a habitualized understanding of what she is looking for. She then performs a meta-level assessment of the topic by reading a book's blurb, followed by a content-level assessment of the writing style to determine if the book matches the desired reading experience. This process can be repeated until one or several books with the desired criteria are located. Note that scanning, meta-, and content-level assessment are often applied in sequence, as part of more complex search processes.

Search processes aimed at Topic Discovery or Knowledge \& Understanding are often characterized by more complex and iterative combinations of micro-strategies. The fluidity of such exploratory searches has been described in the context of knowledge work [17, 29, 34, 53] and everyday information seeking [39]. Based on our interviews, we have identified patterns of narrowing and expanding as part of these search processes, similar to elaboration and reduction applied in creative design processes to arrive at the most promising result [27]. For example, when talking about a typical search process in the professional context, one participant described how he iteratively specifies queries as he learns from previous query results: "When I have a topic, and I probably have little idea of what the topic is about, and [when] I start to look for it, I look for the topic in general, and then there are books showing up. So I go through the summaries, so I start to learn something about the topic, and there are a lot of little ideas what I can pick pretty fast. And if I found something what is

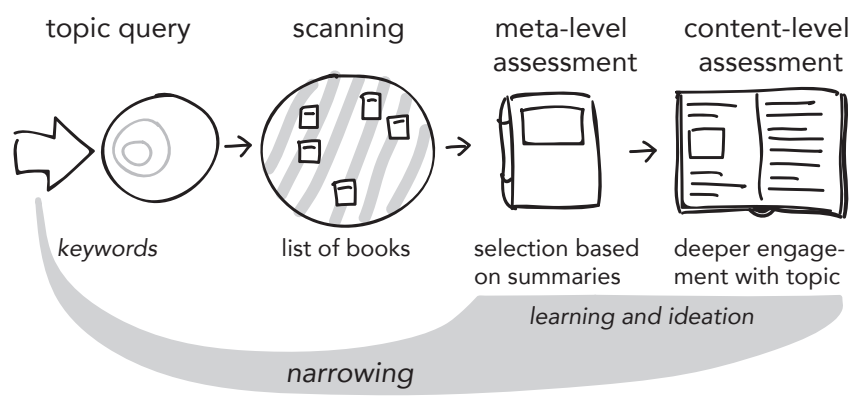

Scenario 2. Iteratively refining and narrowing queries.

more the focus what I have to write about or whatever, then I can go deeper into it, and I can pick other keywords." [R9].

As illustrated in Scenario 2, the search starts with a topic query and scanning of the result list. The following metaand content-level assessment of books leads to ideas for more specific keywords to initiate new queries. This process allows the participant to slowly hone in on his topic of interest.

Other examples we found can be understood as inversions of the previous scenario, where participants started by querying for a particular book, but from there expanded their search by linking to related books by the same author or with related keywords (see Scenario 3). In this process, the purpose of the search changes from a Known Book search to a search by Constrained Criteria, or even for Topic Discovery: "Sometimes I follow, because they have keywords, so if it [the book] is in the subject area and I need to expand my search, I'll put in the specific author and then I'll find out what kinds of areas they are listed under. And then I can click on that link and then find out what else is available. So again it's clicking through the links, that are already supplied." [A9].

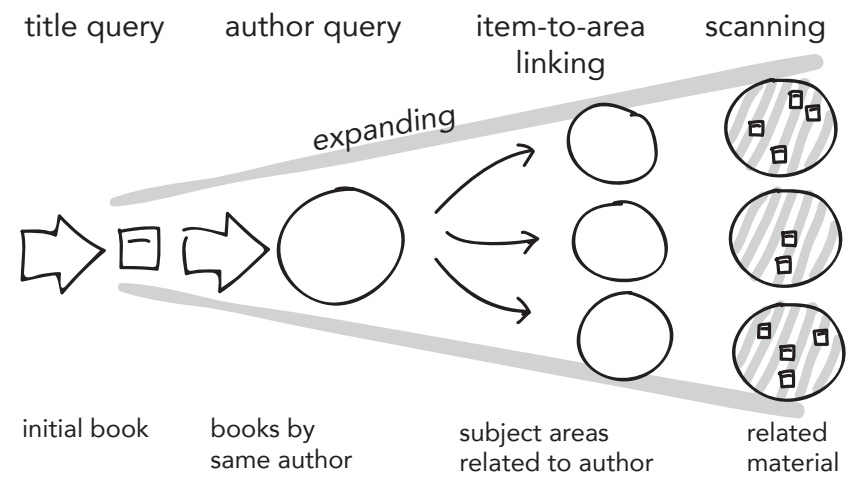

Scenario 3. Expanding the search criteria.

Participants reported on these types of search processes when using digital tools as well as in libraries and bookstores: "I'll have my specific target [when I go to a library] like I said, but then I'll always go and find more stuff as I'm there." [R7]. Search processes in which the result space is expanded by linking to related books or topic areas, and in which the purpose of the search shifts away from the initial target, are often accompanied by serendipitous discoveries. Participants described these search experiences as quite positive: "I appreciate being able to be pleasantly surprised, I appreciate being shown things that I might not otherwise be looking for. 
But I'm also worried of being taken too far off course." [R5]. This statement suggests that search expansions can also be accompanied by hesitation to move too far away from the initial purpose of the search and a fear of getting lost. Interestingly, our participants expressed these negative feelings typically with regard to digital search tools: "[You] get lost in the database and then do not really know where you are as far as what you've searched. It's kind of hard to look back." [A3].

Participants also described types of fluid search processes which they understand as a linear exploration of a book collection on a path from one book to the next (see Scenario 4): "It's like you search for one thing and it leads you [down] a path. And sometimes I spend half an hour looking for something when I found what I needed in the first five minutes, but I ended up continuing, [...] just like seeing what else there was that was related or connected.” [R5].

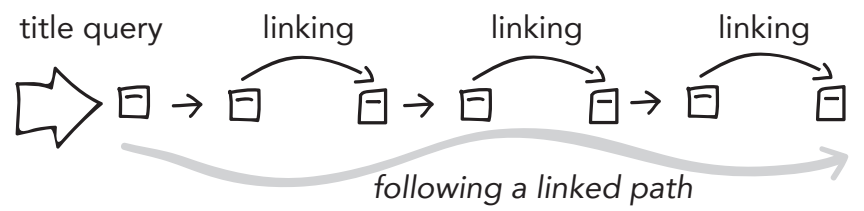

Scenario 4. Following a linked exploration path.

Our findings further demonstrate the creative nature of exploratory search processes including their relation to learning, social aspects, and rewarding experiences.

\section{Search as a Creative Learning Process}

Our analysis shows that iterative and evolving searches [3] are often intertwined with learning and ideation. Assessing search results leads to increasing knowledge about a search topic, which, in turn, results in new ideas on how to refine or expand the search (see Scenarios $2 \& 3$ ). It is the early assessment that aids the learning and ideation process, however, the instantaneous character of digital environments can invite search behaviours that do not include early assessment: "On the internet, I put things into a search engine, [and] I end up following the links. That's usually how I find stuff. The problem with that though is, there are sometimes so many links that I never spend enough time actually looking at the results that come up. [...If] I just stopped and looked at the results and read some more in detail, [...] I would probably get some deeper information and understand it better [...] You click on links because it's so easy. And you end up collecting so many. And sometimes they are just repeats or they aren't actually that useful. And then you just have an overload of information. [...] Electronically things are instantaneous: you can get so much [...] but if you don't process it, that's as good as not getting information at all.” [A9].

In this linear process search and learning are separate. The participant starts her search by querying, scans the results, and collects items for further assessment (see Scenario 5). While she is aware that an early content-level assessment could inspire further explorations and benefit learning, she does not assess the items until the collection is complete. Digital tools encourage this behaviour which leads to an overwhelming amount of potentially irrelevant collected sources.

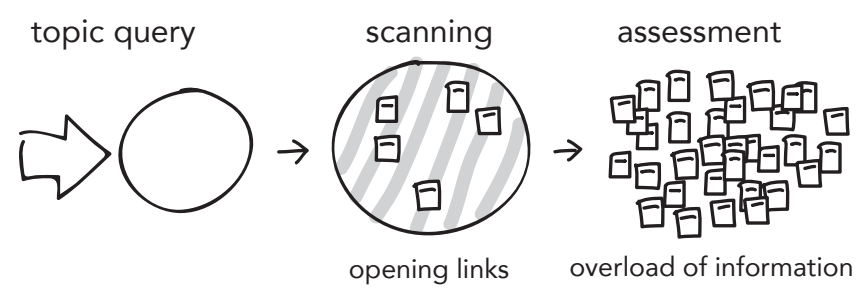

Scenario 5. Separate collecting and learning process.

The same participant stated that, in contrast, in a physical library, her search process is more intertwined with learning (comparable to Scenario 2): "It's the physical weight of things and the physical distance between things that slows you down. That's not a bad thing necessarily. It gives you time to really think about: 'Do I need this information? Is the information useful?' And it forces you to stop and first look at it before you go to the next one. [...] If you are forced to take the physical steps to get to the place and then look at the book and really think about it, then that makes you more efficient than a whole bunch of hits." [A9]. In physical libraries the weight of books practically enforces a content-level assessment as part of the search process, promoting information processing and learning. As the statement indicates, the time that it takes to walk up to bookshelves, physically moving through the information space, is actually appreciated as incubation time that promotes thinking and ideation $[19,28]$. While digital search engines facilitate fast and convenient access to vast information sources, they do not promote content assessment. This comes at the expense of learning and ideation processes.

\section{Social Aspects of Search}

Social influences on exploratory search have been previously mentioned [30, 35, 46, 47, 53]. Similarly, our participants talked about seeking out resources curated by individuals or groups with particular domain knowledge or status: "When I want to find things that are more specific, I usually look for a source, or a collection point, or a blog, or somebody who tweets about it, that I can follow." [R5]. Curated resources are particularly useful as starting points for finding books on specific topics, or defined by "soft" characteristics that are difficult to specify, e.g., concerning a particular reading experience: "I think, what I've done before [is] find a group of people that I know are interested in that type of literature and then I look at their website." [A11]. Recommendations by friends or colleagues are considered most trustworthy and relevant: "I'm usually advised to look at these books, so by my supervisor, or, you know, my friends." [R4].

Annotations in physical books encountered during contentlevel assessment can also enhance search as a social experience: "There is one book in this library, that people have written in, and there is, like, different notes, that people have made. And you can see the hand writing of several different readers-I've even written in it. [...] and that's part of like the heritage of that book." [A9]. Such handwritten annotations make visible how people have explored a book. They leave evidence which information pieces other people found important and help the knowledge building process. 


\section{Search as a Rewarding Experience}

The most open-ended book explorations are driven by the purpose of engaging in a rewarding exploration experience. Participants describe these types of searches as highly opportunistic, serendipitous, and driven by curiosity without implying a particular order of strategies. While generally similar types of micro-strategies are applied, they are carried out with a focus on visual and sensory aspect of the books.

During experience-driven explorations, participants described assessment strategies, such as content skimming and looking at book covers, that are also common in targeted book search. However, in this context, the focus is not on predefined criteria. Instead, books can catch people's attention based on visual aspects: "Sometimes the really funny looking covers are what attracts me in the first place. They don't look like a good book, they are like the two cent cheap novels, but I think that the covers are really funny so I'll take them off the shelf. And there's like, you know, books from the 1920s that have a different feel than the books from 2011. So, I don't know, there is something different about it." [A9]. Here, a book's content or credibility, even the anticipated reading experience is of secondary relevance. Instead, the experience value lies in its visual appearance (e.g., a "funny cover" [A9]), or its physical appeal or even smell: "What I like is the smell of a new book." [R9]. The exploration experience itself takes priority and applied criteria opportunistically form, evolve, and change as part of the exploration.

In searches when the main purpose is a pleasurable and inspiring exploration experience, the visual presentation of a book collection plays an important role. A layout that enables scanning and meta-level assessment of visual and tangible characteristics of books as well as the aesthetic appeal of the search environment can inspire and enhance the exploration experience, as the following statements illustrate: "If you're going through the stacks, it's harder to browse, cause they are, like, closer together, and you have to look closely at the titles. But if they're just laid on a table in front of you it's easy to kind of just scan your eye over them, and then if something catches your eye you grab it. [...] I really like to browse by colour, that's something that I feel like, when I'm at the library for real. I'm looking at different colours, things that catch my eye. [...] It's like aesthetically pleasing, it's kind of nice to look at, so you want to explore that more." [R7].

Our interviews indicate that physical spaces offer a more appealing environment for pleasurable and rewarding explorations. In addition to their visual richness, libraries and bookstores provide manageable collection sizes and a sense of continuity. This makes the exploration experience comfortable and rewarding by aiding navigation while avoiding the feeling of being overwhelmed or "getting lost" [A3]-a common experience in digital information spaces. "When I look for books I mainly, since it [the physical space] is categorized by sections, it's really easier to find where you are, what you are looking for. For instance, I look for, I don't know, books about music and books about, let's say, cooking even. And then I, since it's a targeted area, I know that all they have is here. So either I find it here or not." [R4].

\section{DESIGNING MODULAR SEARCH INTERFACES}

Our findings show the fluidity in which micro-strategies are composed into complex search processes. Now, how does the deconstruction of search and knowledge about the composition of search processes that are driven by creativity, ideation, and learning inform the design of digital search interfaces? As discussed earlier, a number of valuable high- and lowlevel guidelines for designing digital search interfaces already exist $[8,21,24,35,32,46,51,53,56]$. We revisit and synthesize these with the modularity of search processes in mind. If search is composed of the four micro-strategies we propose in this paper, this can directly inform the design of versatile search interfaces that promote creativity and learning. As Makri and Warwick argue, "it is possible to support creativity, albeit indirectly, by ensuring that electronic resources are designed to support the lower level behaviours [...] in ways that might lead to creativity" [32, p.1767].

Supporting Individual Micro-Strategies. As our search scenarios show, querying is often integrated in iterative search processes where the goals are ill-defined. It is therefore important to support dynamic querying $[47,53]$, query by example [21], and a tight coupling of the query and results [35].

When it comes to displaying a collection of books-be it before or after a query has been issued-it is important to visually highlight different characteristics and/or facets of the collection. This can promote thinking by free association which, in turn, can support ideation, creativity, and learning during search processes [46]. This aspect in particular must be considered when designing for linking. Digital search interfaces can provide a range of different types of links, and thus present a "well-connected space and navigational aids" that supports browsing [26]. The key is to make these linking branches visible to guide the exploration [46]. Inspired by research on information-based ideation [24], we suggest providing visual previews of the thematic area behind a link to promote curiosity and foreshadow directions of potential exploration paths, which can further minimize the fear of getting lost in the collection. As Kerne et al. suggest, the presentation of rich (visual) links within and across collections can also help to provide stimuli to overcome fixation or getting stuck [24].

Visual overviews of a subset or the entire book collection are also important to facilitate scanning. Whitelaw suggests "generous interfaces", in which rich collection overviews are provided without requiring people to issue a query first [54]. Along these lines, websites such as Pinterest have been found to promote creativity and ideation [30]. Visual overviews can be item-based or include abstract visualizations [35, 51] to inspire new exploration paths. In assessment strategies, characteristics of the individual book and its content play an important role [8]. Making high-level information about a book available, as well as its content (including keyword searches within the book), can improve assessment [53, 54] and thus integrate learning into the search process.

Fluidity Through Continuous Navigation. Our findings show that a sense of continuity is a valuable aspect when exploring physical information environments. It is important to main- 
tain the context and avoid abrupt switches between different search stages/views. In digital search, participants expressed anxiety over "getting lost" when navigating away from result lists to item views, which is generally detrimental to creative thinking and ideation [28]. Furthermore, a disconnect of assessment strategies from the overall search process (see Scenario 5) compromises the integration of learning and search. Related work suggests easing the transition from overviews to detailed perspectives as well as displaying resources in context of their collection [13]. Enabling continuous navigation supports the fluid combination of micro-strategies. If screen real estate permits, such fluid transitions can be realized by providing all-in-one views of the collection/results, item meta-data, and previews. Other more compact solutions include layering these components so that a sense of presence of the collection/result list is provided at all times; even during the examination of a single item.

Histories and Exploration Trails. Our research confirms the importance of search histories [38, 46, 53] to encourage exploration and fostering people's understanding of an information space or a collection, while minimizing the fear of losing focus or getting lost. One way of doing this is to make exploration paths that lead to a particular item explicit, for instance, through visual breadcrumbs, illustrating queries or highlighting visited links. While common search websites support the "browser history" feature, visualization-based interfaces often do not. Supporting this type of history can increase peoples willingness to explore beyond initial search goals, enable sharing results, and consult previous searches at a later point in time to find more related or similar material.

Offering Curated Collections. Curated sub-collections can provide starting points for less directed, experience-oriented explorations or for searches with hard-to-define criteria (e.g., a particular reading experience). The support of knowledge sharing has been recommended in the context of collaboration and creativity [46, 53]; the curation of content can be considered as beneficial for creative ideation [30, 24, 38]. Integrating such features from digital creative communities ${ }^{1}$ where people make collections of hand-picked items available for others to browse into search interfaces can help to support the creative aspects and outcomes of search. The key here is to present such items in visual rather than textual ways, which provides a rich overview into the content the item represents.

Playfulness and Rich Interaction. Playful representation and interaction approaches to digital collections promote explorations where the experience is a reward in itself. In physical environments people engage in sensory experiences that are driven by visual cues and curious discoveries. Previous research highlights the importance of supporting serendipity $[16,51]$ through unexpected juxtaposition of items [26, 51] and by enticing curiosity and promoting playful explorations [51]. Play has also been discussed as an important facilitator for creativity and inspiration [41, 52]. More concretely, this can be achieved by highlighting outliers, by supporting organic animations and transitions, and by providing rich visual cues that invite discovery and further exploration.

\footnotetext{
${ }^{1}$ https://www.etsy.com/; http://www.pinterest.com/
}

Furthermore, digital technology, such as direct-touch displays can promote a playful approach to information exploration.

\section{CONCLUSION}

We have presented an in-depth qualitative analysis of people's book search habits in professional and personal contexts and in a large spectrum of digital and physical book search environments. Our findings reflect that book search can be considered as a complex process that is constructed from four basic micro-strategies: querying, linking, scanning, and assessing. Depending on the search purpose, these processes can take on highly unique and diverse forms where creativity, ideation and learning are an outcome and driver of the search process. As such social, sensory, and visual aspects play an important role in rewarding information explorations. Based on these insights, and by revisiting and synthesizing previous research on search and creativity, we have introduced a modular perspective on search. Our work informs the design of search interfaces that promote and facilitate creative and versatile search endeavours. Future research will investigate the generalizability of our model beyond book search.

\section{ACKNOWLEDGEMENTS}

We would like all our study participants for sharing their approaches to search with us. We also thank Jagoda Walny and Lindsay MacDonald who provided useful feedback on this paper. Last but not least we thank our funding agencies-NSERC, SurfNet, AITF, Grand, Smart Technologies - that made this research possible.

\section{REFERENCES}

1. Bates, M. J. Information Search Tactics. Journal of American Society for Information Sciences 30 (1979), 205-214.

2. Bates, M. J. An exploratory paradigm for online information retrieval. In Proc. of Information Science (1985), 91-99.

3. Bates, M. J. The design of browsing and berrypicking techniques for the online search interface. Online Information Review 13, 5 (1989), 407-431.

4. Beghetto, R. A., and Kaufman, J. C. Toward a Broader Conception of Creativity: A Case for "mini-c" Creativity. Psychology of Aesthetics, Creativity, and the Arts 1, 2 (2007), 73-79.

5. Boyatzis, R. Transforming Qualitative Information: Thematic Analysis and Code Development. Sage Publications, 1998.

6. Buchanan, G., Cunningham, S. J., Blandford, A., and Rimmer, J. Information Seeking by Humanities Scholars. In Research and advanced technology for digital libraries. 2005, 218-229.

7. Buchanan, G., and Loizides, F. Investigating document triage on paper and electronic media. In Research and Advanced Technology for Digital Libraries, vol. 4675 LNCS. 2007, 416-427.

8. Buchanan, G., and Mckay, D. In the Bookshop : Examining Popular Search Strategies. Proc. of JCDL'11 (2011), 269-278.

9. Chang, S.-J., and Rice, R. E. Browsing: A Multidimensional Framework. Annual review of information science and technology (1993), 231-276.

10. Chang, S.-J. L. Information Research in Leisure: Implications from an Empirical Study of Backpackers. Library Trends 57, 4 (2009), 711-728.

11. Choo, C. W., B, D., and Turnbull, D. Information Seeking on the web: An integrated model of browsing and searching. First Monday 5, 2 (2000), 1-13.

12. Dörk, M., Carpendale, S., and Williamson, C. The information flaneur: A fresh look at information seeking. In Proc. of CHI'11 (2011), 1215-1224. 
13. Dörk, M., Carpendale, S., and Williamson, C. Fluid views: a zoomable search environment. In Proc. of AVI (2012), 233-240.

14. Ellis, D. A behavioural approach to information retrieval design. Journal of Documentation 45, 3 (1989), 171-212.

15. Fidel, R. Moves in Online Searching. Online Review 9, 1 (1985), 61-74.

16. Ford, N., Miller, D., Alan, O., Ralph, J., Turnock, E., and Booth, A. Information Retrieval and creativity: Towards support for the original thinker. Journal of Documentation 55, 5 (1999), 528-542.

17. Foster, A. A nonlinear model of information-seeking behavior. Journal of the American Society for Information Science and Technology 55, 3 (Feb. 2004), 228-237.

18. Fulton, C. The pleasure principle: the power of positive affect in information seeking. Proc. of Aslib 61, 3 (2009), 245-261.

19. Gabora, L. Cognitive Mechanisms Underlying the Creative Process. In Proc. of Creativity and Cognition (2002), 126-133.

20. Hartel, J. Information activities and resources in an episode of gourmet cooking. Information Research 12, 1 (2006), 54-57.

21. Hearst, M. A. Search User Interfaces. Cambridge University Press, 2009

22. Kari, J., and Hartel, J. Information and Higher Things in Life : Addressing the pleasurable and the profound in information science. Journal of the American Society for Information Science and Technology 58, 8 (2007), 1131-1147.

23. Kaufman, J. C., and Beghetto, R. a. Beyond big and little: The four c model of creativity. Review of General Psychology 13, 1 (2009), 1-12.

24. Kerne, A., Webb, A. M., Smith, S. M., Linder, R., Lupfer, N., Qu, Y., Moeller, J., and Damaraju, S. Using Metrics of Curation to Evaluate Information-Based Ideation. ACM Transactions on Computer-Human Interaction 21, 3 (2014), 1-48.

25. Kuhlthau, C. C. Inside the search process: Information seeking from the user's perspective. Journal of the American Society for Information Science 42, 5 (June 1991), 361-371.

26. Kwasnik, B. H. A Descriptive Study of the Functional Components of Browsing. In Proc. of Engineering for $\mathrm{HCI}$ (1992), 191-203.

27. Laseau, P. Graphic Thinking for Architects and Designers. John Wiley and Sons, 1980.

28. Lee, S.-S., Theng, Y.-L., and Goh, D. H.-L. Creative information seeking Part I: a conceptual framework. Proc. of Aslib 57, 5 (2005), 460-475.

29. Lee, S.-S., Theng, Y.-L., and Goh, D. H.-L. Creative information seeking: Part II: empirical verification. Proc of Aslib 59, 3 (2007), 205-221.

30. Linder, R., Snodgrass, C., and Kerne, A. Everyday ideation: all of my ideas are on pinterest. In Proc. of CHI'14 (2014), 2411-2420.

31. Makri, S. A Study of Lawyers' Information Behaviour Leading to the Development of Two Methods for Evaluating Electronic Resources. PhD thesis, University College London, 2009.

32. Makri, S., and Warwick, C. Information for Inspiration: Understanding Architects Information Seeking and Use Behaviors to Inform Design. Journal of the American Society for Information Science and Technology 61, 9 (2010), $1745-1770$.

33. Marchionini, G. Information-seeking strategies of novices using a full-text electronic encyclopedia. Journal of the American Society for Information Science 40, 1 (1989), 54-66.

34. Marchionini, G. Information seeking in electronic environments. Cambridge University Press, 1997.
35. Marchionini, G. Exploratory Search: From finding to understanding. Communications of the ACM 49, 4 (2006), 41-46.

36. Marchionini, G., and White, R. W. Find What You Need, Understand What You Find. Journal of Human-Computer Interaction 23, 3 (2008), 205-237.

37. Marshall, C. C., and Shipman, F. M. Spatial hypertext and the practice of information triage. Proc of HYPERTEXT '97 (1997), 124-133.

38. m.c. Schraefel. Building Knowledge: What's beyond Keyword Search? IEEE Computer 42, 3 (2009).

39. McKenzie, P. J. A model of information practices in accounts of everyday-life information seeking. Journal of Documentation 59, 1 (2003), 19-40.

40. Mikkonen, A., and Vakkari, P. Readers' Search Strategies for Accessing Books in Public Libraries. In Proc. of Information Interaction in Context (2012).

41. Russ, S. W. Affect and creativity: The role of affect and play in the creative process. Psychology Press, 1993.

42. Savolainen, R. Everyday life information seeking: Approaching information seeking in the context of "way of life". Library \& Information Science Research 17, 3 (1995), 259-294.

43. Sheldrick Ross, C. Finding without seeking: the information encounter in the context of reading for pleasure. Information Processing \& Management 35, 6 (Nov. 1999), 783-799.

44. Sheldrick Ross, C. Making Choices: What Readers Say About Choosing Books to Read for Pleasure. The Acquisitions Librarian 13, 25 (2000), 5-21.

45. Shneiderman, B. Clarifying search: A user-interface framework for text searches. D-Lib Magazine (1997).

46. Shneiderman, B. Creating creativity: user interfaces for supporting innovation. ACM Transactions on Computer-Human Interaction 7, 1 (2000), 114-138.

47. Shneiderman, B. Creativity Support Tools: Accelerating Discovery and Innovation. Communications of the ACM 50, 12 (2007), 20-32.

48. Smith, A. G. Internet search tactics. Online Information Review 36, 1 (2012), 7-20.

49. Spiller, D. The provision of fiction for public libraries. Journal of Librarianship and Information Science 12, 4 (1980), 238-266.

50. Sutcliffe, A., and Ennis, M. Towards a cognitive theory of information retrieval. Interacting with Computers 10 (1998), 321-351.

51. Thudt, A., Hinrichs, U., and Carpendale, S. The Bohemian Bookshelf: Supporting Serendipitous Discoveries through Information Visualizaiton. In Proc. of CHI'12 (2012), 1461-1470.

52. Vandenberg, B. Play, problem-solving, and creativity. New Directions for Child and Adolescent Development 1980, 9 (1980), 49-68.

53. White, R. W., and Roth, R. A. Exploratory Search: Beyond the Query-Response Paradigm. Synthesis Lectures on Information Concepts, Retrieval, and Services 1, 1 (Jan. 2009), 1-98.

54. Whitelaw, M. Towards Generous Interfaces for Archival Collections. Presented at ICA, Brisbane, 2012.

55. Wildemuth, B. M., Oh, J. S., and M, G. Tactics Used When Searching for Digital Video. In Proc. of Information Interaction in Context (2010).

56. Wilson, M. Search-User Interface Design. Morgan \& Claypool Publishers, 2011. 